\title{
Efecto de la ganancia de peso invernal sobre el desarrollo genital de vaquillas a los 18 meses
}

\author{
Rouvier, A.M.'; Yáñez, E.A. ${ }^{2}$ \\ ${ }^{1}$ Actividad privada, Provincia de Chaco, Argentina. ${ }^{2}$ Profesor Departamento de Producción Animal, Facultad de \\ Ciencias Veterinarias (FCV), Universidad Nacional del Nordeste (UNNE), Cabral 2139, Corrientes, Argentina. \\ E-mail: enriquey.mv@gmail.com
}

\begin{abstract}
Resumen
Rouvier, A.M.; Yáñez, E.A.: Efecto de la ganancia de peso invernal sobre el desarrollo genital de vaquillas a los 18 meses. Rev. vet. 28: 2, 93-98, 2017. La edad de primer servicio en vaquillas es una de las grandes limitantes en el nordeste argentino. El objetivo de este trabajo fue determinar el efecto de diferentes ganancias de peso vivo en el primer invierno en confinamiento, con recría estival en pastizal natural, sobre el crecimiento, desarrollo corporal y score genital en vaquillas a los 18 meses de edad. Para el ensayo se utilizaron 45 terneras de tipo Brangus y en la primer parte del estudio se las distribuyó en 3 corrales, asignándoles cantidades diferentes de la dieta: tratamiento ad-libitum (T1, ganancia esperada $800 \mathrm{~g} /$ día), tratamiento con $15 \%$ de restricción (T2), y tratamiento con $30 \%$ de restricción (T3), continuando luego todas juntas en pastoreo. Las variables medidas fueron altura a la grupa (AG), perímetro torácico $(\mathrm{PT})$, peso vivo $(\mathrm{PV})$, grasa subcutánea de cadera (P8) y score genital (SG). El diseño fue completamente al azar con tres tratamientos y 15 repeticiones. Los resultados obtenidos en T1 fueron AG 116,6 y 131,3 cm; PT de 153,3 y 158,6 cm; PV de 290 y 301,2 kg; P8 de 5,85 y $3,76 \mathrm{~mm}$; SG de 2,80 y 3,23 , para el final del período de confinamiento y final del período pastoril, respectivamente. Estos resultados permiten afirmar que los animales se encontraban en condiciones de recibir el primer servicio. A los 18 meses, las vaquillas T2 no presentaron diferencias con T1 en ninguno de los parámetros evaluados. El T3 fue diferente de T1 en todas las variables, excepto en el SG, para el cual no se observaron diferencias estadísticas, atribuible a la gran variabilidad de los datos. La restricción del $15 \%$ de la dieta en el período de recría invernal a corral no afectó el desarrollo corporal ni genital de las vaquillas.
\end{abstract}

Palabras clave: vaquilla, crecimiento, score genital, recría,servicio, condición corporal.

\begin{abstract}
Rouvier, A.M.; Yáñez, E.A.: Winter weight gain effect over the genital development in 18 month old heifers. Rev. vet. 28: 2, 93-98, 2017. The age for first service in cows is challenging considering breeding conditions for the northeast region of Argentina. The purpose of this work was to determine the effect of different live weight gains (PV) during the first winter in confinement, with natural grassland in summer rearing, on growth, body development and genital score (SG) in eighteen month old cows. For this assay Brangus calves $(n=45)$ were used. During the first part of the assay animals were distributed in three lots and food was administered in different amounts: ad-libitum treatment expected weight gain $800 \mathrm{~g} /$ days (T1); $15 \%$ food restriction treatment (T2), and 30\% food restriction (T3). Later all animals continued grazing altogether. Variables under consideration were PV, SG, hip height, thoracic perimeter, and subcutaneous fat from hip (P8). Design was completely randomized with 3 treatments and 15 repetitions. Results for $\mathrm{T} 1$ at the end of confinement and grazing period were: hip height 116.6 and $131.3 \mathrm{~cm}$; thoracic perimeter 153.3 and $158.6 \mathrm{~cm}$; PV 290 and $301.2 \mathrm{~kg}$; P8 5.85 and $3.76 \mathrm{~mm}$; and SG 2.80 and 3.23 . These results confirm that they were able to receive the first service. The T2 animals did not show differences compared to T1 for none of the evaluated parameters. $\mathrm{T} 3$ was different compared to $\mathrm{T} 1$ in all the variables, except for genital score in which statistic differences were not observed being this due to large data variability. The $15 \%$ food restriction during winter did not affect heifers body and genital development.
\end{abstract}

Key words: heifers, growth, genital score, body condition. 


\section{INTRODUCCIÓN}

Una de las principales limitantes productivas y económicas de los sistemas de cría que se desarrollan en la región del nordeste argentino (NEA), es la edad con que las vaquillas alcanzan su primer entore y la respuesta reproductiva al mismo, motivada por la falta de buen desarrollo al inicio de la misma ${ }^{18}$.

Los recursos forrajeros predominantes del NEA son las pasturas estivales, caracterizadas por presentar dos etapas marcadamente diferentes: período primavero-estival, de desarrollo acelerado, cuando se logran ganancias diarias de peso vivo (GDPV) que permiten obtener buenos ritmos de crecimiento (aproximadamente $500 \mathrm{~g}$ ), y otoño-invernal en el cual las gramíneas tropicales se encuentran maduras, obteniéndose GDPV nulas ${ }^{8} \mathrm{e}$ incluso pérdidas de peso vivo (PV). La amplia heterogeneidad en su composición y potencial productivo en distintos ambientes, estaciones y años, hacen necesaria su complementación para mejorar el aprovechamiento y la respuesta animal.

Durante la recría la vaquilla debe mantener un ritmo de crecimiento que le permita alcanzar prontamente la pubertad. La aptitud reproductiva se establece como consecuencia del desarrollo corporal y de los órganos reproductivos ${ }^{19}$. El momento en que las vaquillas alcancen la pubertad condicionará el porcentaje de preñez y la distribución de la misma durante su primer servicio.

El objetivo en dicha etapa es lograr un ritmo de crecimiento que permita alcanzar el desarrollo y condición corporal (CC) adecuados para obtener alta eficiencia reproductiva en el primer entore, que contribuya a evitar la disminución del porcentaje de preñez en el segundo servicio. Una baja tasa de crecimiento de los animales implica que pasen a servicio vaquillonas de escaso tamaño corporal (comprometiendo su vida reproductiva), o que sea necesario esperar que las mismas tengan tres años para realizar su primer servicio (incrementando los costos).

En el primer invierno post-destete se presenta el más importante desbalance nutricional que limita la productividad de los bovinos jóvenes. Contrastan en ese período altos requerimientos proteicos y energéticos de las terneras en crecimiento, con el bajo valor nutritivo del campo natural. Una estrategia para solucionar este problema, es realizar la recría durante el primer invierno, con una alimentación a corral y ganancias moderadas, con expectativa de crecimiento compensatorio en la posterior etapa a campo.

El fundamento que justifica la regulación de la ganancia de PV en el corral es el crecimiento compensatorio, definido como el rápido incremento en la tasa de crecimiento relativo a la edad exhibido por animales alimentados en forma adecuada a sus requerimientos, luego de un período de restricción nutricional suficiente para deprimir el desarrollo continuo ${ }^{15}$. Los animales mayores a 6 meses de edad son menos sensibles a la se- veridad de la restricción y exhiben una mejor respuesta compensatoria ${ }^{11}$.

La explicación fisiológica del crecimiento compensatorio estaría vinculada a una reducción metabólica producida en los animales restringidos ${ }^{11}$, cuyos efectos se prolongarían en la fase de realimentación, con utilización más eficiente de los nutrientes. A la reducción metabólica se le suman el aumento en el consumo, al mayor eficiencia de conversión, el cambio en la composición de los tejidos y factores endocrinos ${ }^{16}$.

Si bien el ternero es la categoría de máxima eficiencia de conversión, por lo cual logra la mayor ganancia en el corral, sería beneficioso el uso del crecimiento compensatorio que permitiría maximizar el margen bruto de la actividad, así como el ingreso neto de la empresa. Para mejorar la competitividad de la cría en campo natural frente a la invernada, el confinamiento de terneros es una buena estrategia ${ }^{24}$.

No se encuentra información suficiente sobre cuál es la GDPV adecuada en una etapa de alimentación de alto costo (corral en invierno), que puede ser compensada en una etapa posterior de abundancia de forraje de bajo costo (primavera-verano). Debido a esto, se fijó como objetivo del presente trabajo determinar el efecto de diferentes ganancias de PV en el primer invierno en confinamiento, con recría estival en pastizal natural, sobre el crecimiento, desarrollo corporal y score genital de vaquillas a los 18 meses de edad.

\section{MATERIAL Y MÉTODOS}

La experiencia se realizó con vaquillas tipo Brangus nacidas en octubre del año 2011, que se identificaron con media señal y caravanas numeradas, en el establecimiento Don Adolfo S.A., campo de pastizales naturales ubicado al sureste de la Provincia del Chaco, Argentina, sobre ruta provincial número 49, $\mathrm{km} \mathrm{30,} \mathrm{lu-}$ gar donde fueron criadas hasta su destete.

En mayo de 2012, una semana antes del destete, se procedió a la marcación, identificación con caravanas de trazabilidad y aplicación de sanidad preventiva. Luego del destete y tratamiento con antiparasitario, fueron trasladadas al establecimiento Doña Lucía, ubicado en Basail (Chaco, Argentina), sobre la ruta nacional $11 \mathrm{~km}$ 946, de la misma empresa. A los 10 días se completó el plan sanitario preventivo.

Realizado el acostumbramiento a la alimentación en comederos durante 15 días, para formar los lotes fueron seleccionadas 45 terneras homogéneas en peso y tipo, con PV promedio de $158 \pm 3,5 \mathrm{~kg}$. Estos animales se distribuyeron al azar en 3 grupos de 15 animales cada uno, y fueron alojadas en corrales con superficie de $20 \mathrm{~m}^{2}$ por ternera, contando con un metro de comedero y $20 \mathrm{~cm}$ lineales de bebedero por animal.

Se comenzó a ofrecer la dieta correspondiente dividida en dos suministros diarios, a las 8 y $16 \mathrm{~h}$ respectivamente. Las terneras fueron alimentadas con dieta formulada con silo de planta entera de sorgo, expeller de girasol, grano de maíz y núcleo mineral, cuya com- 
posición químico-bromatológica fue determinada por el laboratorio de la empresa Teknal SA. La composición se ajustó para terneras britanizadas, con una GDPV esperada de 800 g/día (Tabla 1).

Los tratamientos fueron: T1-ad libitum (animales a los que se suministró la dieta al 3\% del PV en materia seca), T2-restricción 15\% (animales a los que se suministró el $85 \%$ de la dieta de T1) y T3-restricción 30\% (animales a los que se suministró el 70\% de la dieta de T1).

Estos tratamientos se aplicaron a partir del 28/6/2012 y hasta el $13 / 12 / 2012$. Luego los animales retornaron a su establecimiento de origen, donde continuaron todos juntos su recría a campo, pastoreando pastizal natural en un potrero de cañada reservado.

Al inicio del experimento se determinó el PV, altura a la grupa y perímetro torácico, repitiendo las determinaciones cada 30 días, tanto durante el confinamiento como en la etapa pastoril. Para el PV se empleó una balanza mecánica de $3500 \mathrm{~kg}$ y para las restantes variables una cinta métrica inextensible.

A los 13 meses de vida (edad en la que se dio por finalizada la etapa de confinamiento) y a los 18 meses -cuando concluyó el trabajo experimental- se monitoreó por palpación transrectal la evolución del tracto reproductivo mediante score genital (SG) utilizando la escala de 1 a $5^{1}$, complementado con ecografía ovárica transrectal para determinar tamaño folicular y presencia de cuerpo lúteo. Se determinó también la CC, evaluada en la escala de puntuación de 1 a $9^{7}$.

Otra variable que se evaluó a los 13 y 18 meses de edad de las vaquillas fue P8, una medida ecográfica de grasa subcutánea de cadera. El P8 fue medido a nivel de la grupa, en el punto de intersección de los músculos Gluteus medius y Biceps femoris ${ }^{22}$. Previa limpieza del lugar, se determinó el sitio de toma de imagen mediante palpación y se usó aceite comestible para favorecer el contacto del transductor, utilizando un stand-off de material siliconado para asegurar el empalme.

Las mediciones ecográficas se realizaron con un ecógrafo Falco (Pie Medical), empleando transductor de 6-8 Mhz, y los resultados se obtuvieron utilizando el software disponible en dicho aparato.

Se utilizó un diseño completamente al azar, con tres tratamientos (ad libitum, 15\% restricción, 30\% restricción) y 15 repeticiones. La unidad experimental la constituyó cada animal. Los datos se analizaron mediante análisis de varianza (ANOVA) y posterior prueba de comparación de medias por Tukey $(\alpha=0,05)$, para todas las variables evaluadas. Los análisis se realizaron con el software estadístico Infostat ${ }^{4}$.

\section{RESULTADOS Y DISCUSIÓN}

El PV (Tabla 2) respondió con aumento constante, con excepción de T3, que durante las primeras etapas del encierre mostró pérdida de $\mathrm{PV}$, pero al terminar el confinamiento obtuvo GDPV de $0,30 \mathrm{~kg} / \mathrm{dí}$.

Las vaquillas $\mathrm{T} 2$ no presentaron pérdida de PV pero tampoco lograron las ganancias previstas en las primeras etapas. Luego de 45 días estos animales alcanzaron la GDPV esperada (500 g/día) y al salir del encierre no presentaban diferencias con las vaquillas T1 (dieta ad libitum) con una formulación para ganancia de $800 \mathrm{~g} /$ día, considerada óptima para la recría ya que el sobreengrasamiento en esta etapa no es aconsejable.

En la etapa en campo natural se observó que las vaquillas T1 fueron las que más sufrieron la adaptación a este ambiente, con importante pérdida de PV en el primer mes en pastoreo $(<19 \mathrm{~kg})$, lo que podría ser adjudicado a varias causas: a) tipo de pastizales disponible ya que es una época del año donde el crecimiento del pastizal es explosivo, perdiendo calidad rápidamente; b) estrés térmico propio de esta época del año, y c) mayor requerimiento de mantenimiento por caminar en suelos encharcados.

El grupo T2, si bien disminuyó su PV al inicio, perdió solamente $12 \mathrm{~kg}$, a diferencia de los animales T3, que redujeron $1 \mathrm{~kg}$ de $\mathrm{PV}$ promedio en ese período.

Una vez superada esta etapa, que podría llamarse de acostumbramiento al pastoreo, los animales de los tres tratamiento empezaron a ganar PV logrando los tratamientos 1 y 2 alcanzar los dos tercios del PV de las vacas adultas de ese rodeo, es decir que estaban en condiciones de ser entoradas. La Figura 1 muestra la evolución de la GDPV para los tres tratamientos, notándose las pérdidas de PV de la etapa inicial a campo.

El estatus nutricional y su consecuente tasa de crecimiento influyen en la aparición de la pubertad ${ }^{9}$. 


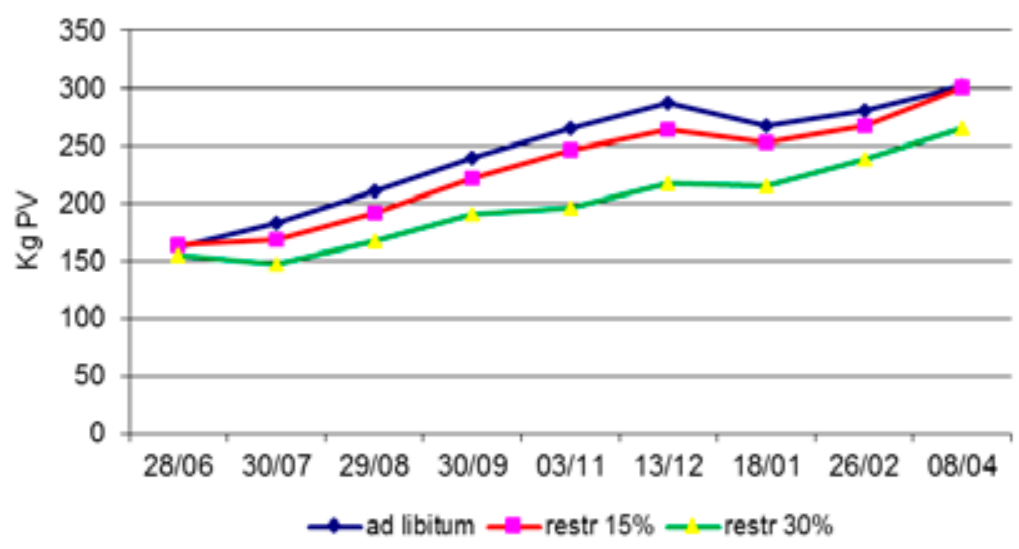

Figura 1. Evolución del peso vivo de las vaquillas de los tres tratamientos, durante todo el ensayo (etapas de confinamiento y pastoril), entre el 28/6/2012 y el 08/04/2013.

Otros autores coincidieron con este concepto al demostrar que las vaquillas con ganancias diarias de PV de $0,50 \mathrm{~kg}$, exhibían incrementos en la frecuencia de descarga de LH y alcanzaban la pubertad más tempranamente que aquellas con ganancias de $0,25 \mathrm{~kg}^{6}$. Las vaquillas con alta tasa de ganancia son más pesadas y de menor edad al inicio de la pubertad, que las vaquillas con bajas ganancias ${ }^{23}$.

El aumento del PV incide fuertemente sobre el inicio de la pubertad y es una variable de predicción para la aparición de la misma. Otros investigadores, utilizando un modelo de regresión logística, revelaron que se comporta como variable de ajuste y marca un umbral crítico de $222 \mathrm{~kg}$ al inicio del segundo invierno, y utilizando regresión múltiple y análisis de componentes principales, concluyeron que el PV es una variable de ajuste y que las vaquillas más pesadas a los 15 meses, entraban en pubertad a una edad menor ${ }^{2}$.

Se determinó que $330 \mathrm{~kg}$ en primavera es el PV crítico (umbral) en vaquillas Brahman y cruza Brahman $\mathrm{x}$ Hereford $(2 / 3 \mathrm{~B}: 1 / 3 \mathrm{H})$, para disponer de una proporción importante de animales sexualmente activos $(80 \%)^{21}$. En este trabajo los animales alcanzaron $300 \mathrm{~kg}$ de PV con $30 \%$ de animales sexualmente activos, observando que este ensayo finalizó en una fecha $(08 / 04)$ en la cual las horas luz disminuyen con un posible efecto negativo sobre la ciclicidad de las vaquillas. Otra investigación plantea un PV umbral más elevado $(350 \mathrm{~kg})$ para lograr un $80 \%$ de vaquillas acebuzadas ciclando, mientras que en vaquillas pampizadas lograron un 90\% ciclando con $300 \mathrm{~kg}^{20}$.

Debería considerarse si las vaquillas presentarían mayor ciclicidad si la evaluación se hubiera realizado 30 o 45 días antes, aún con menor PV. Hay que considerar también que en este estudio las vaquillas tenían 18 meses y período en pastoreo. $(\mathrm{p}<0,05)$. no 24 meses de edad como en los trabajos citados.

El comportamiento de la variable circunferencia torácica en todo el período fue muy similar a lo observado con el PV, confirmando que son dos parámetros directamente correlacionados. Como se observa en la Tabla 3, al comenzar el periodo a corral no había diferencia entre tratamientos, debido a la homogeneidad del lote. A la salida del confinamiento se observan diferencias significativas entre tratamientos y luego del periodo en pastoreo, se marca el crecimiento compensatorio de los tratamientos con restricciones, llegando las vaquillas T2 a alcanzar el perímetro de las T1. Si bien en los animales T3 se observó crecimiento compensatorio, el mismo no fue suficiente para alcanzar el mismo desarrollo.

La altura a la grupa es uno de los parámetros más convenientes para describir el tamaño corporal en vacas de carne ${ }^{2}$. En un rodeo de vaquillas Nelore ubicado en el norte del estado de San Pablo, Brasil, 275 a $320 \mathrm{~kg}$ fue el PV alcanzado para el servicio con 2 años de edad, con 132 a $138 \mathrm{~cm}$ de altura y 6,5 puntos de $\mathrm{CC}^{13}$. Estos resultados son superiores a los encontrados en nuestro ensayo para las variables en estudio, destacando que esos autores trabajaron con animales de mayor edad así como también con estructura corporal (frame) superior.

En vaquillas Brahman x Hereford púberes de 14 meses ${ }^{27}$ se describen valores de altura a la grupa de 119 cm y PV de $272 \mathrm{~kg}$, inferiores a los observados en este trabajo. Por otra parte en vaquillas Brahman x Hereford de 2 años se reportaron valores de $125 \mathrm{~cm}$ de altura a la grupa ${ }^{17}$, similares a los aquí obtenidos (Tabla 4).

Trabajando con vaquillas Angus, un investigador constató que de las variables ecográficas de composi-

Tabla 3. Promedios de circunferencia torácica en $\mathrm{cm}(\overline{\mathrm{X}} \pm \mathrm{DE})$ durante el inicio del estudio, la salida del confinamiento y la finalización del

\begin{tabular}{lccc}
\hline tratamiento & $28 / 06 / 2012$ & $13 / 12 / 2012$ & $08 / 04 / 2013$ \\
\hline ad libitum & $130,3 \pm 5,2 \mathrm{a}$ & $158,0 \pm 6,8 \mathrm{a}$ & $158,6 \pm 5,9 \mathrm{a}$ \\
restricción 15\% & $129,2 \pm 6,7 \mathrm{a}$ & $153,2 \pm 7,8 \mathrm{a}$ & $159,0 \pm 7,5 \mathrm{a}$ \\
restricción 30\% & $127,7 \pm 7,5 \mathrm{a}$ & $142,0 \pm 7,5 \mathrm{~b}$ & $149,9 \pm 5,6 \mathrm{~b}$ \\
\hline
\end{tabular}

Valores en la misma columna con distintas letras son diferentes entre sí

Tabla 4. Altura a la grupa $(\mathrm{cm})$ durante el inicio del estudio, la salida del confinamiento y la finalización del período en pastoreo $(\overline{\mathrm{X}} \pm \mathrm{DE})$.

\begin{tabular}{lccc}
\hline tratamiento & $28 / 06 / 2012$ & $13 / 12 / 2012$ & $08 / 04 / 2013$ \\
\hline ad libitum & $106,8 \pm 3,1 \mathrm{a}$ & $119,2 \pm 3,5 \mathrm{a}$ & $125,4 \pm 3,6 \mathrm{a}$ \\
restricción 15\% & $107,9 \pm 2,9 \mathrm{a}$ & $118,3 \pm 2,7 \mathrm{a}$ & $125,3 \pm 2,6 \mathrm{a}$ \\
restricción 30\% & $105,3 \pm 2,6 \mathrm{a}$ & $114,2 \pm 3,8 \mathrm{~b}$ & $120,4 \pm 3,0 \mathrm{~b}$ \\
\hline
\end{tabular}

Valores en la misma columna con distintas letras son diferentes entre sí $(\mathrm{p}<0,05)$. 
Tabla 5. Grasa subcutánea de cadera ( $\mathrm{P} 8$, en $\mathrm{mm})$, a la salida del confinamiento y al finalizar el período en pastoreo ( $\overline{\mathrm{X}} \pm \mathrm{DE})$.

\begin{tabular}{lcc}
\hline tratamiento & $13 / 12 / 2012$ & $08 / 04 / 2013$ \\
\hline ad libitum & $5,85 \pm 1,57 \mathrm{c}$ & $3,76 \pm 1,11 \mathrm{~b}$ \\
restricción $15 \%$ & $4,13 \pm 1,27 \mathrm{~b}$ & $3,92 \pm 1,20 \mathrm{~b}$ \\
restricción $30 \%$ & $2,33 \pm 0,74 \mathrm{a}$ & $2,55 \pm 0,58 \mathrm{a}$ \\
\hline
\end{tabular}

Valores en la misma columna con distintas letras son diferentes entre sí $(\mathrm{p}<0,05)$.

Tabla 6. Score genital ( $\overline{\mathrm{x}} \pm \mathrm{DE})$ al final del confinamiento y al final de la etapa pastoril.

\begin{tabular}{lcc}
\hline tratamiento & $13 / 12 / 2012$ & $08 / 04 / 2013$ \\
\hline ad libitum & $2,80 \pm 0,94 \mathrm{~b}$ & $3,23 \pm 0,73 \mathrm{a}$ \\
restricción $15 \%$ & $2,73 \pm 0,96 \mathrm{~b}$ & $3,00 \pm 0,88 \mathrm{a}$ \\
restricción 30\% & $1,79 \pm 0,70 \mathrm{a}$ & $2,60 \pm 0,91 \mathrm{a}$ \\
\hline
\end{tabular}

Valores en la misma columna con distintas letras son diferentes entre sí $(\mathrm{p}<0,05)$.

ción corporal, la única con capacidad predictiva fue el P8 ${ }^{12}$, observando que el PV era una variable significativa para predecir la edad a la pubertad, pero ni la GD (grasa dorsal) ni el AOB (área ojo de bife) pudieron ser predictores al respecto. Sin embargo en ese trabajo, el P8 tuvo la tendencia a constituir una variable valiosa en el modelo para la predicción de la pubertad. Coincidiendo con esto, existe una fuerte hipótesis de que son necesarios niveles críticos de grasa y proteína para el inicio de la pubertad ${ }^{3}$.

Se informaron valores de $\mathrm{P} 8 \mathrm{de} 4 \mathrm{~mm}$ en vaquillas cruza y Angus, de GD de 2,5 mm en las cruzas y 3,2 $\mathrm{mm}$ en las Angus, así como de AOB entre 21 y $22 \mathrm{~cm}^{2}$ para ambos biotipos ${ }^{5}$. Estos datos de P8 son levemente mayores a los obtenidos en este trabajo al final del ensayo (Tabla 5), en tanto que a la salida del confinamiento los valores que se obtuvieron fueron superiores para $\mathrm{T} 1$ y T2.

Se considera que el PV y la masa del tejido adiposo juegan un rol crítico en la regulación del inicio de la pubertad ${ }^{10}$, en tanto que otros autores encontraron una fuerte correlación genética entre las mediciones ecográficas ( $\mathrm{P} 8, \mathrm{GD}$ y $\mathrm{AOB}$ ) en el animal vivo al servicio y sus medidas equivalentes en la carcasa ${ }^{28}$. Por lo tanto, la selección a través de la mejoría en la composición de la carcasa de las vacas, puede tener un impacto sobre la fertilidad de su descendencia.

Las mediciones ecográficas son también utilizadas en el animal vivo para relacionarlas con la madurez de las vaquillas ${ }^{12}$. Esto fue confirmado por autores que analizaron la relación entre la madurez del tracto reproductivo (SG, escala de Anderson) y las variables ultrasonográficas de composición corporal (AOB, GD, y P8) y el PV. Ellos observaron que las vaquillas más pesadas y con mayor grasa en la grupa tuvieron mayor grado de desarrollo reproductivo al servicio (SG: 4 y 5) ${ }^{1}$.

En vaquillas cruza cebú se encontraron valores de SG de 3,3 puntos asociados a $323 \mathrm{~kg}$ de PV y CC de 5 al momento de aparición de la pubertad ${ }^{5}$. En el presente trabajo se logró prácticamente el mismo SG en el tratamiento ad libitum y algo menor en el T2 (Tabla 6), con hembras que presentaron CC: 5 al realizarse el SG.

A su vez los resultados observados coinciden en general con lo informado en lotes de vaquillas cruza cebú con CC entre 4 y 4,5 puntos y PV de 277; 281; 296 y $318 \mathrm{~kg}$, que presentaron SG de 2; 3; 4 y 5 puntos respectivamente ${ }^{26}$.

Estos datos muestran que las vaquillas con menor $\mathrm{PV}$ que las empleadas de este ensayo y menor $\mathrm{CC}$ al momento de realizar la medición, presentaban SG de 4 puntos, lo que permite inferir que las vaquillas de los tratamientos con mejor desempeño no presentaron mayor SG por la época del año en que se realizó la evaluación.

Lotes de vaquillas cruza cebú con pesos de 282; 292; 300; 310 y $338 \mathrm{~kg}$ presentaron un SG de 1; 2; 3; 4 y 5 puntos respectivamente ${ }^{25}$. Los resultados del presente trabajo son coincidentes, ya que a medida que aumenta el PV de las vaquillas, se incrementa el SG. Las vaquillas con peso $\geq 300 \mathrm{~kg}$ presentan $\mathrm{SG}$ entre 4 y 5 puntos.

En conclusión, las vaquillas alimentadas ad libitum y restrictas en un $15 \%$ durante el período de recría a corral, llegaron a los 18 meses sin tener diferencias en ninguna de las variables estudiadas y en condiciones de ser entoradas por primera vez.

Se puede afirmar que en la recría con una etapa de confinamiento y otra pastoril, se puede gastar un 15\% menos en los costos de alimentación en la etapa de confinamiento, mejorando así la rentabilidad del sistema.

Agradecimientos. A la empresa Establecimientos Don Adolfo SA por el aporte de animales, instalaciones, alimentación y personal. Al equipo de reproducción de la EEA INTA Colonia Benítez, por las determinaciones ecográficas, y en particular a la Dra. Noelia Prieto por sus aportes.

\section{REFERENCIAS}

1. Anderson KJ, Le Fever DG, Brinks JS, Odde KG. 1991. The use of reproductive tract scoring in beef heifers. AgriPractice 12: 4-19.

2. Baker JF, Stewart TS, Long CR, Cartwright T. 1988. Multiple regression and principal components analysis of puberty and growth in cattle. J Anim Sci 66: 2147-2158.

3. Brooks AL, Morrow RE, Youngquist RS. 1985. Body composition of beef heifers at puberty. Theriogenology 24 : 235-250.

4. Di Rienzo JA, Casanoves F, Balzarini MG, González L, Tablada M, Robledo CW. 2010. Grupo InfoStat, FCA, UNC, Argentina.

5. Evans HL, Willard ST, King R, Vann RC. 2004. Case study: relationships among live animal carcass traits, the estrous cycle, and synchronization of estrus in beef heifers. The Prof Anim Scient 20: 453-459. 
6. Hall JB, Schillo KK, Fitzgerald BP, Bradley NW. 1994. Effects of recombinant bovine somatotropin and dietary energy intake on growth, secretion of luteinizing hormone, follicular development, and onset of puberty in beef heifers. J Anim Sci 72: 709-718.

7. Herd DB, Sprott LR. 1990. Body condition, nutrition and reproduction of beef cows. Agri-life communications and marketing, Publ. The Texas A\&M University System. http://agrilifebookstore.org

8. Holgado F. 2008. Entore anticipado de la vaquillona, Informe Técnico INTA Leales (Tucumán, Argentina). http:// inta.gob.ar/sites/default/files/script-tmp-entoreant.pdf

9. Kinder JE, Bergfeld EG, Wehrman ME, Peters KE, Kojima F. 1995. Endocrine basis for puberty in heifers and ewes. J Reprod Fertil Suppl.49: 393-407.

10. Lassek WD, Gaulin SJ. 2007. Menarche is related to fat distribution (brief communication). Am J Phys Anthrop 133: 1147-1151.

11. Lawrence TL, Fowler VR. 1997. Growth of farm animals, CAB International, University Press, Cambridge, UK, 330 p.

12. Marambio M. 2005. The use of ultrasound body composition measurements to track puberty in heifers and to predict the reproductive ability of mature beef cows. Thesis of Master of Science, Ohio State University, $66 \mathrm{p}$.

13. Mercadante ME, Packer IU, Razook AG, Cyrillo JN, Figueiredo L. 2003.Direct and correlated responses to selection for yearling weight on reproductive performance of Nelore cows. J Anim Sci 81: 376-384.

14. Minick JA, Wilson DE, Rouse GH, Hassen A, Sealock MP, Hopkins S. 2001. Relationship between body composition and reproduction in heifers. Beef Research Report, Iowa State University, Ames. http://lib.dr.iastate.edu/beefreports_2001/35

15. Ojeda A, Molina F, Carmona D. 2007. Crecimiento compensatorio: una estrategia de manejo de la disponibilidad de pasturas. XI Seminario de manejo y utilización de pastos y forrajes en sistemas de producción animal, UCLA, Barquisimeto (Venezuela), http://www.avpa.ula.ve/eventos/xi_seminario/Conferencias/Articulo-4.pdf

16. Olazábal LJ, San Martín HF. 2008. Crecimiento compensatorio. Publ. Univ Nac San Marcos, Perú. http://veterinaria.unmsm.edu.pe/files/SIRIVS\%20JO.pdf

17. Patterson DJ et al. 1991. Evaluation of reproductive traits in Bos taurus and Bos indicus crossbred heifers: effects of post weaning energy manipulation. J Anim Sci 69: 23492361 .
18. Patterson DJ, Perry RC, Kiracofe GH, Bellows RA, Staigmiller RB, Corah LR. 1992. Management considerations in heifer development and puberty. J Anim Sci 70: 4018-4035.

19. Patterson DJ, Wood SL, Randle RF. 2000. Procedures that support reproductive management of replacement beef heifers J Anim Sci 77: 1-15.

20. Pourrain A, Beckwith B. 1993. Peso corporal crítico para el primer entore de vaquillas cruza Brahman x Hereford. En: Evaluación y elección de biotipos de acuerdo a los sistemas de producción, Memorias XXXV, IICA-Procisur, Montevideo. Uruguay, p. 265-267.

21. Pourrain A. 2001. Los biotipos en el ganado vacuno. La importancia de conocer sus características y comportamiento. Noticias y Comentarios (Publ. Estación Experim. Agropec. Mercedes, Corrientes, Argentina), ISSN 03273059, No 357, p. 6.

22. Realini CE, Williams RE, Pringle T, Bertrand J. 2001. Gluteus medious rump fat depths as additional live animal ultrasound measurements for predicting retail product and trimmable fat in beef carcasses. J Anim Sci 79: 1378-1385.

23. Short RE, Bellows RA. 1971. Relationships among weight gains, age at puberty and reproductive performance in heifers. J Anim Sci 32: 127-131.

24. Simeone A. 2007. Suplementación energética y proteica en condiciones de pastoreo. En Seminario sobre suplementación y engorde a corral, Young, Río Negro, Argentina, p. 57-86.

25. Stahringer R, Maidana G, Suarez L. 2001.Efecto de dos esquemas de administración de GnRH y prostaglandina en la sincronización de celo de vaquillas cruza cebú con distinto grado de desarrollo genital. Reunión Com. Cient. Técnol. 2001, UNNE (Corrientes). http://www1.unne.edu. ar/cyt/2001/4-Veterinarias/V-022.pdf.

26. Stahringer R, Mastandrea O. 2005. Respuestas de vaquillas cruza cebú con distinto grado de desarrollo genital a la sincronización de celo con progestágenos, GnRh y/o prostaglandina. http://www.produccion-animal.com.ar/

27. Stewart TS, Long CR, Cartwright TC. 1980. Characterization of cattle of a five-breed diallel. III: Puberty in bulls and heifers. J Anim Sci 50: 808-820.

28. Wilson DE, Rouse GE, Hays CL. 2001. Carcass EPDs from Angus heifer real-time ultrasounds scans. Iowa State University Beef Research Report ASL-R1736, Ames. 5 p. http://lib.dr.iastate.edu/beefreports_2001 